...of the

patients who had undergone RYGB surgery, those that experience postprandial hypoglycaemia had a higher than normal variability in their glucose levels...

\title{
SURGERY
}

\section{Postprandial hypoglycaemia following bariatric surgery}

Bariatric surgery is known to be associated with intractable and debilitating postprandial hypoglycaemia, but the mechanisms for this association remain unclear. A new study published in the European Journal of Endocrinology has begun to answer this question.

"Previous studies on postprandial hypoglycaemia in patients who have undergone bariatric surgery have shown mixed and sometimes contradictory findings, to some extent due to poor standardization of methodologies and variable performance from hormone assays," explains corresponding author Tricia Tan. "In our study we assessed our patients in the most comprehensive way possible, using multiple techniques to determine glycaemic variability and gut hormone responses to a meal."

The study included 37 individuals (28 who had undergone Roux-en-Y gastric bypass (RYGB) surgery and 9 obese controls) from the Imperial Weight Centre, London, UK. To evaluate glycaemic variability, Tan and colleagues used continuous glucose monitoring and a standardized mixed-meal test to compare gut hormone responses in patients who had postprandial hypoglycaemia with those who did not.

Tan and colleagues found that of the patients who had undergone RYGB surgery, those that experience postprandial hypoglycaemia had a higher than normal variability in their glucose levels. Interestingly, the authors also found that glucagon and glucagon-like peptide 1 (GLP1) were secreted in excess during the early part of the mixed-meal test, before the development of hypoglycaemia.

"Both glucagon and GLP1 are insulinotropic, and the combination of both leads to excessive insulin secretion and therefore hypoglycaemia," explains Tan. "Endocrinologists typically think of glucagon as the hormone that is secreted after hypoglycaemia to boost blood glucose levels, but we found that the glucagon was being secreted before hypoglycaemia occurred. We speculate that this might be because both GLP1 and glucagon are being secreted by the L cells in the gut following stimulation by food entering the small intestine."

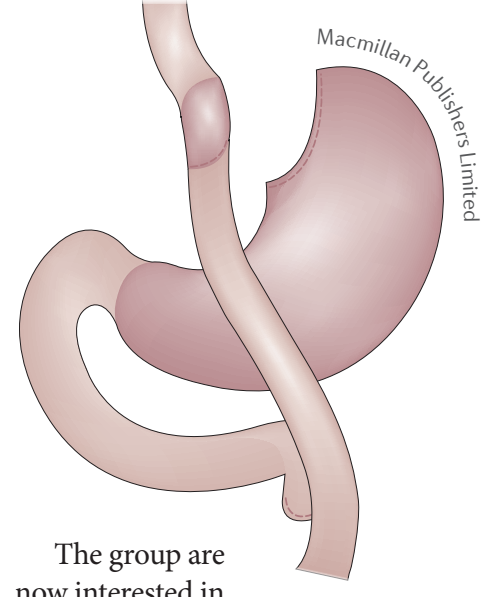

now interested in confirming some promising data that suggest that exendin 9-39 (a GLP1 antagonist) can help treat postprandial hypoglycaemia. They are also investigating whether specific dietary changes can help patients who experience postprandial hypoglycaemia following bariatric surgery.

Alan Morris

ORIGINAL ARTICLE Tharakan, G. et al. Roles of increased glycemic variability, GLP-1 and glucagon in hypoglycaemia after Roux-en-Y gastric bypass. Eur.J. Endocrinol. http://dx.doi. org/10.1530/EJE-17-0446 (2017) 